\title{
Multiple mechanisms contribute to the development of clinically significant azole resistance in Aspergillus fumigatus
}

\section{W. S. Moye-Rowley*}

Department of Molecular Physiology and Biophysics, Carver College of Medicine, University of lowa, lowa City, IA, USA

\section{Edited by:}

Frederic Lamoth, Duke University, USA

\section{Reviewed by:}

Laura Alcazar-Fuoli, Instituto de Salud Carlos III, Spain

Alix Thérèse Coste, University Hospital of Lausanne, Switzerland

*Correspondence:

W. S. Moye-Rowley, Department of Molecular Physiology and Biophysics, Carver College of Medicine, University of lowa, 6-530 Bowen

Science Building, lowa City, IA 52242, USA

e-mail: scott-moye-rowley@uiowa.edu
Infections caused by the filamentous fungus Aspergillus fumigatus are a significant clinical issue and represent the second most-common form of fungal infection. Azole drugs are effective against this pathogen but resistant isolates are being found more frequently. Infections associated with azole resistant $A$. fumigatus have a significantly increased mortality making understanding drug resistance in this organism a priority. The target of azole drugs is the lanosterol $\alpha-14$ demethylase enzyme encoded by the cyp51A gene in $A$. fumigatus. Mutations in cyp51A have been described that give rise to azole resistance and been argued to be the primary, if not sole, contributor to azole resistance. Here, I discuss recent developments that indicate multiple mechanisms, including increased expression of ATP-binding cassette $(A B C)$ transporter proteins, contribute to azole resistance. $A B C$ transporters are well-established determinants of drug resistance in other fungal pathogens and seem likely to play a similar role in A. fumigatus.

\section{Keywords: Aspergillus fumigatus, ABC transporter, azole resistance, cyp51A, abcG1}

\section{INTRODUCTION}

Antibiotics have been one of medical science's greatest advances but their continued efficacy is at risk. Over prescription and improper use has led to dramatic increases in resistant microorganisms with accompanying increased mortality. The problem of increased resistance is especially acute for fungal pathogens as the basic eukaryotic cell biology of these organisms limits the classes of antibiotics that avoid effects on the host.

Aspergillus fumigatus is the primary filamentous fungal pathogen of humans and an especially serious issue in any immunocompromised situation (Ruping et al., 2008). Azole drugs are most commonly deployed against $A$. fumigatus (and most fungi) as these drugs are well-tolerated and can be orally delivered. The success of azole antifungal therapy has naturally led to their extensive use both in the clinical setting and agriculturally, especially in Europe (discussed in Verweij et al., 2009). Strikingly, azole antifungals have been much more limited in their application in the United States (Pham et al., 2014) and this seems likely to help explain the differences between resistance mechanisms seen in these two regions. Increased azole resistance is a serious issue in treatment of aspergillosis. Mortality of azole-resistant aspergillosis can approach $90 \%$ (van der Linden et al., 2011).

Although the first azole resistant isolates of A. fumigatus were reported in the United States (Denning et al., 1997), understanding of the molecular basis of resistance was led by experiments from Europe. Early work on azole resistant isolates of A. fumigatus determined that these organisms contained mutations in one of the two genes $(c y p 51 \mathrm{~A} / \mathrm{B})$ encoding the azole target enzyme: lanosterol $14 \alpha$ demethylase (Mellado et al., 2001). Focus on this gene (cyp51A) led to the surprising finding that the overwhelming majority of azole resistant strains contained two mutations and that both alterations were required for azole resistance (Verweij et al., 2007). This allele is referred to as TR34/L98H and consists of a $34 \mathrm{bp}$ duplication in the promoter region linked to a replacement of leucine 98 with a histidine residue in the sequence of the enzyme (Mellado et al., 2007). This cyp51A allele confers multiazole resistance and has been found in resistant organisms ranging from Europe to India (Snelders et al., 2008; Chowdhary et al., 2012).

The origin of the TR34/L98H resistance allele as an environmentally selected variant is supported by several circumstantial lines of evidence. First, isolates from the soil led to the alarming discovery that $5-7 \%$ of these A. fumigatus strains contain this multiazole resistance lesion (reviewed in Chowdhary et al., 2013). Second, patients not previously exposed to azole drugs also exhibit aspergillosis with the TR34/L98H-containing fungus (Mellado et al., 2007). Third, genomic analyses of multiazole resistant A. fumigatus determined that strains containing the TR34/L98H allele were the most closely related compared to other azole-susceptible organisms, consistent with these drug resistant strains emerging from a single and recent alteration (Camps et al., 2012b). Finally, study of azole resistant organisms in the United States, where agricultural use of azoles is dramatically lower, has not uncovered the prevalence of the TR34/L98H cyp51A variant in drug resistant isolates (Pham et al., 2014).

While there is no question $c y p 51 \mathrm{~A}$ mutations are important contributors to azole resistance, more recent studies have provided evidence that other resistance mechanisms are also at work in A. fumigatus (reviewed in Vermeulen et al., 2013). These other mechanisms have been observed in isolates derived from patients chronically exposed to azole drugs. This long term challenge with 
azole drugs drives generation of mutant spores that are now drug resistant. Since A. fumigatus exists in a multicellular state, acquisition of a mutant allele in one nucleus is very unlikely to produce a resistant organism. However, if a mutant spore is generated, then a resistant isolate will be produced. Analyses of these mutant strains of A. fumigatus that have been recovered after azole exposure have uncovered multiple new mechanisms of resistance to these antifungal drugs. Here I will review recent experiments that implicate the participation of ATP-binding cassette (ABC) transporter proteins and other mechanisms in azole resistance of clinical isolates.

\section{ABC TRANSPORTERS AND DRUG RESISTANCE IN PATHOGENIC FUNGI}

The predominance of the TR34 L98H cyp51A allele in A. fumigatus represents an uncommon genetic distribution of azole resistance in other, better understood fungi. The pathogenic fungus for which we have the most detailed understanding of azole resistance mechanisms is the major human pathogen Candida albicans. Extensive analyses of the molecular basis for azole resistance in C. albicans led to the discovery of at least two different routes. The C. albicans ERG11 gene encodes the lanosterol $14 \alpha$ demethylase in this fungus. Changes both in the sequence of the protein as well as alterations that increase ERG11 transcription are associated with azole resistance (Perea et al., 2001; Morio et al., 2010). Increased expression of membrane transporter proteins is a second mechanism that synergizes with ERG11 changes (reviewed in Morschhauser, 2010). Experiments in different clinical isolates (Selmecki et al., 2008; MacCallum et al., 2010) led to the finding that high level azole resistance required the cooperation of both the ERG11 gene and a transcription factor encoded by the TAC1 gene. The key role of Tacl is to induce expression of ABC transporter proteins encoded by the CDR1 gene along with other targets (Coste et al., 2004). These transporter proteins are of two different functional classes: the ABC transporters and major facilitator superfamily (MFS) transporters (reviewed in Prasad and Goffeau, 2012; Costa et al., 2014). Typically, these membrane proteins are found in the plasma membrane (PM) where they are thought to act as energy-dependent drug efflux pumps (Marger and Saier, 1993). Overproduction of the membrane transporters is usually due to amino acid substitutions in transcription factors that ultimately drive elevated mRNA level corresponding to these drug pumps.

This theme of elevated ABC transporter expression cooperating with Erg11 to confer azole resistance is seen in other pathogens including Candida krusei (Lamping et al., 2009) and Candida glabrata (Samaranayake et al., 2013). As is usual in instances of drug resistance, these multiple different mechanisms are engaged that work together to produce the full in vivo response to drug challenge. While the synergy of Erg11 and ABC transporters is well-described in Candida species, evidence implicating $\mathrm{ABC}$ transporters and other proteins in A. fumigatus has been less wellappreciated, likely owing in part to the elegant demonstrations tying cyp51A mutations to clinical azole resistance (Snelders et al., 2008). Recent experiments provide a rationale for reconsidering the importance of mechanisms beyond $c y p 51 A$ in azole resistance in A. fumigatus.

\section{AZOLE RESISTANCE IN A. fumigatus MAY UTILIZE NON-cyp51A-DEPENDENT MECHANISMS}

As discussed above, the large environmental reservoir of cyp51A azole resistant organisms has focused much attention on changes in this key target gene giving rise to drug resistant isolates (Verweij etal., 2009). Analyses of A. fumigatus azole resistant isolates from patient populations undergoing chronic azole exposure have provided important new information implicating other resistance pathways in antifungal resistance (Figure 1).

The first detailed study of azole resistant $A$. fumigatus strains that also contained wild-type cyp51A genes came from analyses of fungal isolates submitted to a regional mycology center at Manchester Hospital in the UK (Howard et al., 2009; Bueid et al., 2010). These investigators discovered that, between the years of 20042009, azole resistant isolates increased from 5 to $20 \%$. While another center reported a similar increase in azole resistant $A$. fumigatus isolates (Mortensen et al., 2011), the unique feature of the Manchester data was the presence of a wild-type cyp51A gene in $43 \%$ of these resistant strains. These findings are consistent with more recent data from the United States in which a broad survey of A. fumigatus isolates found an overall lower frequency of azole resistance (5\%) but $98 \%$ of these resistant isolates had no cyp51A lesion known to be associated with azole resistance (Pham et al., 2014). The origin of the A. fumigatus isolates in the UK and US studies were very different with the Manchester patients corresponding to patients with chronic aspergillosis and the US isolates coming from a wide range of sources (Pham et al., 2014). The primary commonality for the resistant isolates from these two different studies was the lack of $c y p 51 A$-dependent changes, strongly indicating the existence of other avenues of azole resistance.

The first data implicating ABC transporters in clinically relevant drug resistance has emerged quite recently. Before discussing these data, I would like to mention the confusing state of current nomenclature for $\mathrm{ABC}$ transporters in A. fumigatus. There is no consensus for gene names in this (or really any) family of protein in Aspergillus and I propose to adopt a system suggested earlier by Kovalchuk and Driessen (2010). This system employs a Human Genome Organization-approved scheme for naming $\mathrm{ABC}$ transporters based on their structural organization. I will use this nomenclature to refer to $\mathrm{ABC}$ transporters from $A$. fumigatus discussed here and encourage others to do the same.

A number of laboratories have demonstrated correlation between increased $\mathrm{ABC}$ transporter expression with associated azole resistance (Tobin et al., 1997; Slaven et al., 2002; Nascimento et al., 2003; da Silva Ferreira et al., 2006) but a functional link between the mRNA levels of a given $\mathrm{ABC}$ transporter and azole resistance remained elusive. Follow-up work on A. fumigatus isolates with a wild-type cyp51A gene led to the finding that mRNA encoding a particular ABC transporter was elevated in 8/11 azole resistant strains (Fraczek et al., 2013). This gene, referred to here as $a b c G 1$ (aka $c d r 1 B$ aka $a b c B$ aka AFUA_1G14330) encodes a homolog of the well-studied ABC transporters Saccharomyces cerevisiae Pdr5 and the Candida species Cdr1. Importantly, disruption of $a b c G 1$ from one of the clinical isolates that overproduced this transcript led to a reduction in itraconazole MIC from $>8$ 


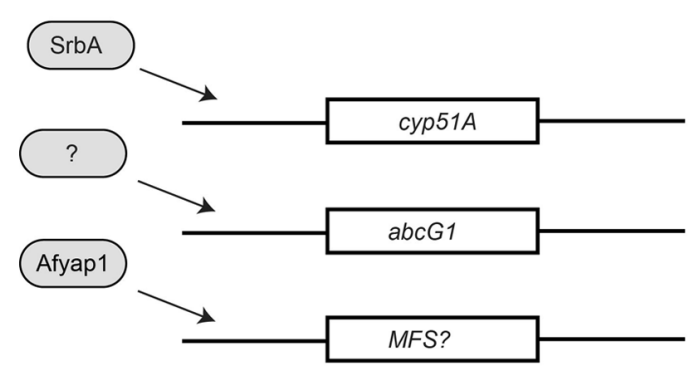

Nucleus

FIGURE 1 | Relationships of azole resistance determinants in Aspergillus fumigatus. Known or putative transcription regulators are indicated in light gray as positive regulators of gene expression. The nuclear genes encoding the lanosterol $\alpha-14$ demethylase (cyp51A), a plasma membrane (PM) ABC transporter (abcG1), or a major facilitator superfamily (MFS) transporter protein are indicated.

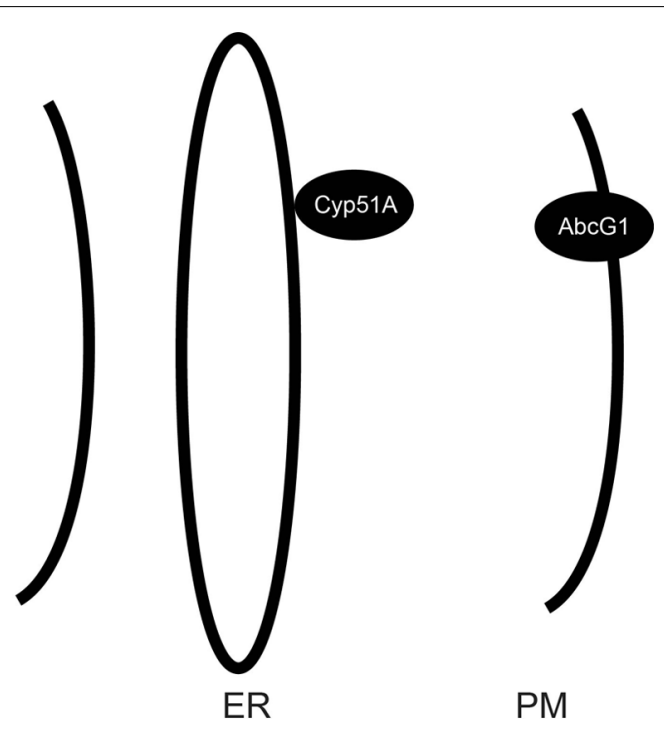

Activation of an MFS-encoding gene by Afyap1 is still speculative at this point but is known to occur in other fungi (Alarco etal., 1997; Coleman etal., 1997; Rognon etal., 2006). The Cyp51A protein is docked to the endoplasmic reticulum (ER) by an $\mathrm{N}$-terminal membrane spanning domain (Monk etal., 2014) while the AbcG1 protein is localized to the PM. to $2 \mathrm{mg} / \mathrm{L}$, demonstrating the functional requirement for this in itraconazole resistance (Fraczek et al., 2013).

Along with this work in clinical isolates, loss of abcG1 was demonstrated to cause profound azole hypersensitivity in four different laboratory strains (Fraczek et al., 2013; Paul et al., 2013). Overproduction of the $a b c G 4$ (aka $c d r 1 A$ aka $a b c A$ aka AFUA_2G15130) gene product, that shares the highest degree of sequence conservation to abcG1, also elevated azole resistance. Fusions of green fluorescent protein to the C-terminus of either abcG1 or abcG4 indicated that the resulting fusion proteins were localized to the PM, consistent with their activity as ATP-dependent drug efflux pumps (Paul et al., 2013). While functional data implicating both of these $\mathrm{ABC}$ transporter proteins has been provided, these types of demonstrations remained to be accomplished for the large number (15) of A. fumigatus ABC transporters in the ABCG class of transporter proteins (Kovalchuk and Driessen, 2010).

While data are accumulating for the role of $\mathrm{ABC}$ transporters in azole resistance in A. fumigatus, other organisms also exhibit drug resistance that is triggered by changes in transcriptional regulators (recently reviewed in Paul and Moye-Rowley, 2014). As mentioned above, C. albicans Tac1 can acquire gain-of-function mutations that lead to enhanced activation of genes under its control (Coste et al., 2004). These target genes include the important azole resistance determinant CDR1. The work of Fraczek et al. (2013) suggests that similar mutations may be present in A. fumigatus azole resistant isolates overproducing $a b c G 1$ but these remain to be found.

However, at least three examples of transcription factors that are important in azole resistance have been described. The first is the sterol response element binding protein (SREBP) from $A$. fumigatus referred to as SrbA (Willger et al., 2008). This regulator was discovered via its sequence conservation with SREBP from Schizosaccharomyces pombe and mammals (reviewed in Bien and Espenshade, 2010). Disruption of the srbA gene led to pronounced azole hypersensitivity, and perhaps most interesting, increased fluconazole susceptibility. This seems likely to be due to the decreased expression of $c y p 51 A$ in the absence of SrbA (Willger et al., 2008) as cyp51A $\Delta$ strains exhibit a similar increased fluconazole susceptibility (Mellado et al., 2005). The importance of the trans-acting factor SrbA in expression of cyp51A suggests the possibility that mutants that increase SrbA activity might elevate azole resistance via their effect on a wild-type cyp51A locus. These type of mutant alleles of $s r b A$ have not been described at present.

A recently discovered azole resistance mechanism involving a transcription factor was described in an isolate from an immunodeficient patient undergoing chronic pulmonary aspergillosis (Camps et al., 2012a). Isolates recovered from early in the infection exhibited an azole susceptible phenotype that ultimately transitioned to azole resistant in time. Clever genetic analyses coupled with whole genome sequencing approaches detected a substitution mutation in the hapE gene as being responsible for the increased azole resistance. A. fumigatus hapE encodes a DNA-binding subunit of the Hap-complex that is a relative of the mammalian CCAAT-binding protein (see Dolfini et al., 2012 for a review). While a mechanistic explanation of the increased azole resistance driven by the mutant HapE-containing complex is still unknown, a potential contributor to this effect is provided by the finding that cyp51A expression is elevated in isolates bearing this lesion (Camps et al., 2012a).

A common link between resistance supported by changes in SrbA or HapE is the potential involvement of cyp51A. Azole 
resistant mutants with changes in these transcription factors could still possess a wild-type cyp51A allele yet exhibit elevated drug resistance. A final example that seems likely to be independent of cyp51A function is provided by the A. fumigatus homolog of $S$. cerevisiae Yap1. This protein is referred to as Afyap1 and is a basic region-leucine zipper transcription factor. ScYap1 is regulated by oxidative stress and normally resides in the cytoplasm due to rapid nuclear export (Kuge et al., 1997; Yan et al., 1998). Upon oxidative stress, nuclear export of ScYap1 is inhibited and the factor accumulates on target promoters, resulting in transcriptional induction of genes involved in redox regulation (reviewed in Morano et al., 2012). Afyap1 has been demonstrated to be regulated by oxidative stress at the level of nuclear localization and seems likely to be controlled by oxidants in a manner similar to that seen for ScYap1 (Lessing et al., 2007).

The relationship of Afyap 1 to azole resistance was demonstrated by work using a truncation form of this protein (Qiao et al., 2010). The carboxy-terminal cysteine-rich domain (c-CRD) was deleted from Afyap 1 and this mutant protein expressed from a multicopy plasmid in A. fumigatus. The c-CRD has been extensively studied in ScYap1 and serves as a negative regulatory site acting to exclude ScYap1 from the nucleus via interaction with the exportin protein Crm1 (reviewed in Guttler and Gorlich, 2011). The Afyap1 mutant lacking its c-CRD (referred to as TR Afyap1) was found to strongly elevate resistance to voriconazole but not influence itraconazole resistance (Qiao et al., 2010). A strain lacking Afyap1 was unaffected in terms of azole resistance but was highly sensitive to oxidants.

This behavior of Afyap1 is quite similar to that previously seen for both the C. albicans Yap1 homolog (Cap1) and ScYap1 (Alarco and Raymond, 1999). Loss of the Yap1-encoding genes from any of these organisms causes oxidative stress hypersensitivity but has no marked influence on azole sensitivity (Alarco et al., 1997; Alarco and Raymond, 1999). However, production of hypermorphic forms of Yap1 homologs does enhance azole resistance, typically through induction of expression of MFS-encoding gene expression (Alarco et al., 1997). Strikingly, in S. cerevisiae, while deletion of the gene encoding the major $\mathrm{ABC}$ transporter involved in azole resistance (Sc PDR5) led to a profound sensitivity to this drug (Sanglard et al., 1995), overproduction of ScYap1 fully suppressed this drug sensitivity (Alarco et al., 1997). This also seems likely to occur in A. fumigatus although further work will be required to confirm this possibility.

\section{SUMMARY}

While early analyses of azole resistance in A. fumigatus were consistent with changes in the cyp51A gene being the primary if not sole driver of drug resistance, recent findings and a more nuanced view of resistance mechanisms do not support this simple picture. The key differentiator between the nearly exclusive involvement of cyp51A mutants in azole resistance and contribution of other pathways comes from consideration of the origin of resistant $A$. fumigatus isolates. An environmental reservoir of resistant organisms, certainly impacted if not completely driven by extensive use of azole-based agricultural fungicides, produced a large number of highly azole resistant isolates that were routinely discovered in patient populations (Verweij et al., 2009). During screening for azole resistant $A$. fumigatus appearing during chronic drug exposure, frequent isolates were found that contained wild-type version of cyp51A (Bueid et al., 2010). These resistant isolates indicate the presence of other resistance pathways in this filamentous fungus as seen in more extensively studied pathogens like the Candida species (Morschhauser, 2002).

The involvement of $\mathrm{ABC}$ transporters in A. fumigatus is most clearly indicated by the studies of Fraczek et al. (2013). Overproduction of $a b c G 1$ was linked to multiple clinical isolates and shown to be required for azole resistance in one. The increasing number of azole tolerant isolates recovered from patients that exhibit no changes in the cyp51A locus supports the view that other mechanisms, such as $\mathrm{ABC}$ transporter overproduction, will be found to play important roles in azole resistance in A. fumigatus.

\section{ACKNOWLEDGMENTS}

I thank Dr. Sanjoy Paul for a critical reading of this manuscript and Dr. Paul Bowyer for helpful discussions. Work on drug resistance in my laboratory is supported by NIH GM49825.

\section{REFERENCES}

Alarco, A. M., Balan, I., Talibi, D., Mainville, N., and Raymond, M. (1997). AP-1-mediated multidrug resistance in Saccharomyces cerevisiae requires FLR1 encoding a transporter of the major facilitator superfamily. J. Biol. Chem. 272, 19304-19313. doi: 10.1074/jbc.272.31.19304

Alarco, A. M., and Raymond, M. (1999). The bZip transcription factor Caplp is involved in multidrug resistance and oxidative stress response in Candida albicans. J. Bacteriol. 181, 700-708.

Bien, C. M., and Espenshade, P. J. (2010). Sterol regulatory element binding proteins in fungi: hypoxic transcription factors linked to pathogenesis. Eukaryot. Cell 9, 352-359. doi: 10.1128/EC.00358

Bueid, A., Howard, S. J., Moore, C. B., Richardson, M. D., Harrison, E., Bowyer, P., et al. (2010). Azole antifungal resistance in Aspergillus fumigatus: 2008 and 2009. J. Antimicrob. Chemother. 65, 2116-2118. doi: 10.1093/jac/dkq279

Camps, S. M., Dutilh, B. E., Arendrup, M. C., Rijs, A. J., Snelders, E., Huynen, M. A., et al. (2012a). Discovery of a HapE mutation that causes azole resistance in Aspergillus fumigatus through whole genome sequencing and sexual crossing. PLoS ONE 7:e50034. doi: 10.1371/journal.pone.0050034

Camps, S. M., Rijs, A. J., Klaassen, C. H., Meis, J. F., O'Gorman, C. M., Dyer, P. S., et al. (2012b). Molecular epidemiology of Aspergillus fumigatus isolates harboring the TR34/L98H azole resistance mechanism. J. Clin. Microbiol. 50, 2674-2680. doi: 10.1128/JCM.00335-12

Chowdhary, A., Kathuria, S., Randhawa, H. S., Gaur, S. N., Klaassen, C. H., and Meis, J. F. (2012). Isolation of multiple-triazole-resistant Aspergillus fumigatus strains carrying the TR/L98H mutations in the cyp51A gene in India. J. Antimicrob. Chemother. 67, 362-366. doi: 10.1093/jac/dkr443

Chowdhary, A., Kathuria, S., Xu, J., and Meis, J. F. (2013). Emergence of azole-resistant Aspergillus fumigatus strains due to agricultural azole use creates an increasing threat to human health. PLoS Pathog. 9:e1003633. doi: 10.1371/journal.ppat.1003633

Coleman, S. T., Tseng, E., and Moye-Rowley, W. S. (1997). Saccharomyces cerevisiae basic region-leucine zipper protein regulatory networks converge at the ATR1 structural gene. J. Biol. Chem. 272, 23224-23230. doi: 10.1074/jbc.272.37. 23224

Costa, C., Dias, P. J., Sa-Correia, I., and Teixeira, M. C. (2014). MFS multidrug transporters in pathogenic fungi: do they have real clinical impact? Front. Physiol. 5:197. doi: 10.3389/fphys.2014.00197

Coste, A. T., Karababa, M., Ischer, F., Bille, J., and Sanglard, D. (2004). TAC1, transcriptional activator of CDR genes, is a new transcription factor involved in the regulation of Candida albicans ABC transporters CDR1 and CDR2. Eukaryot. Cell 3, 1639-1652. doi: 10.1128/EC.3.6.1639-1652.2004

da Silva Ferreira, M. E., Malavazi, I., Savoldi, M., Brakhage, A. A., Goldman, M. H., Kim, H. S., et al. (2006). Transcriptome analysis of Aspergillus fumigatus exposed to voriconazole. Curr. Genet. 50, 32-44. doi: 10.1007/s00294-006-0073-2 
Denning, D. W., Venkateswarlu, K., Oakley, K. L., Anderson, M. J., Manning, N. J., Stevens, D. A., et al. (1997). Itraconazole resistance in Aspergillus fumigatus. Antimicrob. Agents Chemother. 41, 1364-1368.

Dolfini, D., Gatta, R., and Mantovani, R. (2012). NF-Y and the transcriptional activation of CCAAT promoters. Crit. Rev. Biochem. Mol. Biol. 47, 29-49. doi: $10.3109 / 10409238.2011 .628970$

Fraczek, M. G., Bromley, M., Buied, A., Moore, C. B., Rajendran, R., Rautemaa, R., et al. (2013). The cdr1B efflux transporter is associated with noncyp5la-mediated itraconazole resistance in Aspergillus fumigatus. J. Antimicrob. Chemother. 68, 1486-1496. doi: 10.1093/jac/dkt075

Guttler, T., and Gorlich, D. (2011). Ran-dependent nuclear export mediators: a structural perspective. EMBO J. 30, 3457-3474. doi: 10.1038/emboj.20 11.287

Howard, S. J., Cerar, D., Anderson, M. J., Albarrag, A., Fisher, M. C., Pasqualotto, A. C., et al. (2009). Frequency and evolution of Azole resistance in Aspergillus fumigatus associated with treatment failure. Emerg. Infect. Dis. 15, 1068-1076. doi: 10.3201/eid1507.090043

Kovalchuk, A., and Driessen, A. J. (2010). Phylogenetic analysis of fungal ABC transporters. BMC Genomics 11:177. doi: 10.1186/1471-2164-11-177

Kuge, S., Jones, N., and Nomoto, A. (1997). Regulation of yAP-1 nuclear localization in response to oxidative stress. EMBO J. 16, 1710-1720. doi: 10.1093/emboj/16.7.1710

Lamping, E., Ranchod, A., Nakamura, K., Tyndall, J. D., Niimi, K., Holmes, A. R., et al. (2009). Abclp is a multidrug efflux transporter that tips the balance in favor of innate azole resistance in Candida krusei. Antimicrob. Agents Chemother. 53 354-369. doi: 10.1128/AAC.01095

Lessing, F., Kniemeyer, O., Wozniok, I., Loeffler, J., Kurzai, O., Haertl, A., et al. (2007) The Aspergillus fumigatus transcriptional regulator AfYapl represents the major regulator for defense against reactive oxygen intermediates but is dispensable for pathogenicity in an intranasal mouse infection model. Eukaryot. Cell 6, 2290 2302. doi: 10.1128/EC.00267

MacCallum, D. M., Coste, A., Ischer, F., Jacobsen, M. D., Odds, F. C., and Sanglard, D. (2010). Genetic dissection of azole resistance mechanisms in Candida albicans and their validation in a mouse model of disseminated infection. Antimicrob. Agents Chemother. 54, 1476-1483. doi: 10.1128/AAC.01645

Marger, M. D., and Saier, M. H. Jr. (1993). A major superfamily of transmembrane facilitators that catalyse uniport, symport, and antiport. Trends Biochem. Sci. 18 13-20. doi: 10.1016/0968-0004(93)90081-W

Mellado, E., Diaz-Guerra, T. M., Cuenca-Estrella, M., and Rodriguez-Tudela, J. L. (2001). Identification of two different 14-alpha sterol demethylase-related genes (cyp51A and cyp51B) in Aspergillus fumigatus and other Aspergillus species. J. Clin. Microbiol. 39, 2431-2438. doi: 10.1128/JCM.39.7.2431-2438.2001

Mellado, E., Garcia-Effron, G., Alcazar-Fuoli, L., Melchers, W. J., Verweij, P. E., Cuenca-Estrella, M., et al. (2007). A new Aspergillus fumigatus resistance mechanism conferring in vitro cross-resistance to azole antifungals involves a combination of cyp51A alterations. Antimicrob. Agents Chemother. 51, 1897-1904. doi: 10.1128/AAC.01092-06

Mellado, E., Garcia-Effron, G., Buitrago, M. J., Alcazar-Fuoli, L., Cuenca-Estrella M., and Rodriguez-Tudela, J. L. (2005). Targeted gene disruption of the 14alpha sterol demethylase (cyp51A) in Aspergillus fumigatus and its role in azole drug susceptibility. Antimicrob. Agents Chemother. 49, 2536-2538. doi: 10.1128/AAC.49.6.2536-2538.2005

Monk, B. C., Tomasiak, T. M., Keniya, M. V., Huschmann, F. U., Tyndall, J. D., O'connell, J. D., et al. (2014). Architecture of a single membrane spanning cytochrome P450 suggests constraints that orient the catalytic domain relative to a bilayer. Proc. Natl. Acad. Sci. U.S.A. 111, 3865-3870. doi 10.1073/pnas.1324245111

Morano, K. A., Grant, C. M., and Moye-Rowley, W. S. (2012). The response to heat shock and oxidative stress in Saccharomyces cerevisiae. Genetics 190, 1157-1195. doi: 10.1534/genetics.111.128033

Morio, F., Loge, C., Besse, B., Hennequin, C., and Le Pape, P. (2010). Screening for amino acid substitutions in the Candida albicans Erg11 protein of azole-susceptible and azole-resistant clinical isolates: new substitutions and a review of the literature. Diagn. Microbiol. Infect. Dis. 66, 373-384. doi: 10.1016/j.diagmicrobio.2009.11.006

Morschhauser, J. (2002). The genetic basis of fluconazole resistance development in Candida albicans. Biochim. Biophys. Acta 1587, 240-248. doi: 10.1016/S09254439(02)00087-X
Morschhauser, J. (2010). Regulation of multidrug resistance in pathogenic fungi. Fungal Genet. Biol. 47, 94-106. doi: 10.1016/j.fgb.2009.08.002

Mortensen, K. L., Jensen, R. H., Johansen, H. K., Skov, M., Pressler, T., Howard, S. J., et al. (2011). Aspergillus species and other molds in respiratory samples from patients with cystic fibrosis: a laboratory-based study with focus on Aspergillus fumigatus azole resistance. J. Clin. Microbiol. 49, 2243-2251. doi: 10.1128/JCM.0021311

Nascimento, A. M., Goldman, G. H., Park, S., Marras, S. A., Delmas, G., Oza, U., et al. (2003). Multiple resistance mechanisms among Aspergillus fumigatus mutants with high-level resistance to itraconazole. Antimicrob. Agents Chemother. 47, 1719-1726. doi: 10.1128/AAC.47.5.1719-1726.2003

Paul, S., Diekema, D., and Moye-Rowley, W. S. (2013). Contributions of Aspergillus fumigatus ATP-binding cassette transporter proteins to drug resistance and virulence. Eukaryot. Cell 12, 1619-1628. doi: 10.1128/EC. 00171-13

Paul, S., and Moye-Rowley, W. S. (2014). Multidrug resistance in fungi: regulation of transporter-encoding gene expression. Front. Physiol. 5:143. doi: 10.3389/fphys.2014.00143

Perea, S., Lopez-Ribot, J. L., Kirkpatrick, W. R., Mcatee, R. K., Santillan, R. A., Martinez, M., etal. (2001). Prevalence of molecular mechanisms of resistance to azole antifungal agents in Candida albicans strains displaying high-level fluconazole resistance isolated from human immunodeficiency virus-infected patients. Antimicrob. Agents Chemother. 45, 2676-2684. doi: 10.1128/AAC.45.10.2676-2684.2001

Pham, C. D., Reiss, E., Hagen, F., Meis, J. F., and Lockhart, S. R. (2014). Passive surveillance for azole-resistant Aspergillus fumigatus, United States, 2011-2013. Emerg. Infect. Dis. 20, 1498-1503. doi: 10.3201/eid2009.140142

Prasad, R., and Goffeau, A. (2012). Yeast ATP-binding cassette transporters conferring multidrug resistance. Annu. Rev. Microbiol. 66, 39-63. doi: 10.1146/annurev-micro-092611-150111

Qiao, J., Liu, W., and Li, R. (2010). Truncated Afyap1 attenuates antifungal susceptibility of Aspergillus fumigatus to voriconazole and confers adaptation of the fungus to oxidative stress. Mycopathologia 170, 155-160. doi: 10.1007/s11046-010-9309-2

Rognon, B., Kozovska, Z., Coste, A. T., Pardini, G., and Sanglard, D. (2006). Identification of promoter elements responsible for the regulation of MDR1 from Candida albicans, a major facilitator transporter involved in azole resistance. Microbiology 152, 3701-3722. doi: 10.1099/mic.0.29277-0

Ruping, M. J., Vehreschild, J. J., and Cornely, O. A. (2008). Patients at high risk of invasive fungal infections: when and how to treat. Drugs 68, 1941-1962. doi: 10.2165/00003495-200868140-00002

Samaranayake, Y. H., Cheung, B. P., Wang, Y., Yau, J. Y., Yeung, K. W., and Samaranayake, L. P. (2013). Fluconazole resistance in Candida glabrata is associated with increased bud formation and metallothionein production. J. Med. Microbiol. 62, 303-318. doi: 10.1099/jmm.0.044123-0

Sanglard, D., Kuchler, K., Ischer, F., Pagani, J. L., Monod, M., and Bille, J. (1995). Mechanisms of resistance to azole antifungal agents in Candida albicans isolates from AIDS patients involve specific multidrug transporters. Antimicrob. Agents Chemother. 40, 2378-2386. doi: 10.1128/AAC.39.11.2378

Selmecki, A., Gerami-Nejad, M., Paulson, C., Forche, A., and Berman, J. (2008). An isochromosome confers drug resistance in vivo by amplification of two genes, ERG11 and TAC1. Mol. Microbiol. 68, 624-641. doi: 10.1111/j.1365-2958.2008.06176.x

Slaven, J. W., Anderson, M. J., Sanglard, D., Dixon, G. K., Bille, J., Roberts, I. S., et al. (2002). Increased expression of a novel Aspergillus fumigatus ABC transporter gene, atrF, in the presence of itraconazole in an itraconazole resistant clinical isolate. Fungal Genet. Biol. 36, 199-206. doi: 10.1016/S1087-1845(02)00016-6

Snelders, E., Van Der Lee, H. A., Kuijpers, J., Rijs, A. J., Varga, J., Samson, R. A., etal. (2008). Emergence of azole resistance in Aspergillus fumigatus and spread of a single resistance mechanism. PLoS Med. 5:e219. doi: 10.1371/journal.pmed.0050219

Tobin, M. B., Peery, R. B., and Skatrud, P. L. (1997). Genes encoding multiple drug resistance-like proteins in Aspergillus fumigatus and Aspergillus flavus. Gene 200, 11-23. doi: 10.1016/S0378-1119(97)00281-3

van der Linden, J. W., Snelders, E., Kampinga, G. A., Rijnders, B. J., Mattsson, E., Debets-Ossenkopp, Y. J., et al. (2011). Clinical implications of azole resistance in Aspergillus fumigatus, The Netherlands, 2007-2009. Emerg. Infect. Dis. 17, 1846-1854. doi: 10.3201/eid1710.110226 
Vermeulen, E., Lagrou, K., and Verweij, P. E. (2013). Azole resistance in Aspergillus fumigatus: a growing public health concern. Curr. Opin. Infect. Dis. 26, 493-500. doi: 10.1097/QCO.0000000000000005

Verweij, P. E., Mellado, E., and Melchers, W. J. (2007). Multiple-triazole-resistant aspergillosis. N. Engl. J. Med. 356, 1481-1483. doi: 10.1056/NEJMc061720

Verweij, P. E., Snelders, E., Kema, G. H., Mellado, E., and Melchers, W. J. (2009). Azole resistance in Aspergillus fumigatus: a side-effect of environmental fungicide use? Lancet Infect. Dis. 9, 789-795. doi: 10.1016/S1473-3099(09) 70265-8

Willger, S. D., Puttikamonkul, S., Kim, K. H., Burritt, J. B., Grahl, N., Metzler, L. J., et al. (2008). A sterol-regulatory element binding protein is required for cell polarity, hypoxia adaptation, azole drug resistance, and virulence in Aspergillus fumigatus. PLoS Pathog. 4:e1000200. doi: 10.1371/journal.ppat. 1000200

Yan, C., Lee, L. H., and Davis, L. I. (1998). Crmlp mediates regulated nuclear export of a yeast AP-1-like transcription factor. EMBO J. 17, 7416-7429. doi: 10.1093/emboj/17.24.7416
Conflict of Interest Statement: The author declares that the research was conducted in the absence of any commercial or financial relationships that could be construed as a potential conflict of interest.

Received: 20 November 2014; accepted: 20 January 2015; published online: 10 February 2015.

Citation: Moye-Rowley WS (2015) Multiple mechanisms contribute to the development of clinically significant azole resistance in Aspergillus fumigatus. Front. Microbiol. 6:70. doi: 10.3389/fmicb.2015.00070

This article was submitted to Fungi and Their Interactions, a section of the journal Frontiers in Microbiology.

Copyright (C) 2015 Moye-Rowley. This is an open-access article distributed under the terms of the Creative Commons Attribution License (CC BY). The use, distribution or reproduction in other forums is permitted, provided the original author (s) or licensor are credited and that the original publication in this journal is cited, in accordance with accepted academic practice. No use, distribution or reproduction is permitted which does not comply with these terms. 